\title{
O uso do sistema CAD/CAM para confecção de próteses fixas: aplicações e limitações
}

\section{The use of CAD/CAM system in fixed prostheses: applications and limitations}

\section{Luciana Borges ${ }^{1}$ (1) \\ Emilena Maria Castor Xisto Lima ${ }^{2}$ (b) Adriana Oliveira Carvalho 3 (B)}

\begin{abstract}
1Autora para correspondência. Escola Bahiana de Medicina e Saúde Pública (Salvador). Bahia, Brasil. luciana.oborges16@gmail.com ${ }^{2}$ Escola Bahiana de Medicina e Saúde Pública (Salvador), Universidade Federal da Bahia (Salvador). Bahia, Brasil. emilenalima@gmail.com ${ }^{3}$ Escola Bahiana de Medicina e Saúde Pública (Salvador), Universidade Estadual de Feira de Santana (Feira de Santana). Bahia, Brasil.
\end{abstract} aoc1981@hotmail.com

\begin{abstract}
RESUMO | O sistema CAD/CAM, sigla derivada do inglês Computer Aided Design / Computer Aided Manufacturing, é uma tecnologia baseada no desenho de uma estrutura protética em um computador seguida de sua confecção em uma máquina de fresagem. Tem como objetivo a simplificação na produção de estruturas protéticas, otimização do tempo de trabalho e a produção de estruturas com alto padrão de qualidade e estética. O emprego desta tecnologia consiste em três etapas: digitalização do preparo protético, planejamento virtual e produção da restauração. O preparo dentário pode ser digitalizado fora da cavidade oral, sobre o modelo de gesso, ou dentro da cavidade oral, através de um sistema de digitalização intra-oral. Após efetuada a digitalização do preparo, a imagem será transferida para um programa de desenho assistido por computador (CAD), pelo qual o operador poderá desenhar de forma virtual a restauração. Através de uma fresadora ou da impressão digital em 3D, o sistema CAM irá reproduzir a reabilitação desejada. Dependendo da estrutura e do seu objetivo, pode-se optar por diversos materiais, como em resina acrílica, cerâmica, híbrido, metal, e resinas compostas. O objetivo do presente trabalho é explanar o uso do sistema CAD/CAM na Odontologia para confecção de próteses fixas através de um levantamento nas bases de dados Lilacs, Pubmed, Scielo e Google Acadêmico. Pôde-se concluir que a tecnologia CAD/CAM aumenta a versatilidade, a precisão e o custo-benefício da confecção de restaurações indiretas. Acredita-se que em breve, fará parte da rotina clínica da maioria dos cirurgiões-dentistas.
\end{abstract}

PALAVRAS-CHAVE: Computer Aided Design. Prótese dentária. Restauração dentária permanente.

\begin{abstract}
The CAD / CAM system, an acronym derived from the English Computer Aided Design / Computer Aided Manufacturing, is a technology based on the design of a prosthetic structure on a computer followed by its manufacture on a milling machine. It aims at simplifying the production of prosthetic structures, optimizing working time and producing structures with a high standard of quality and aesthetics. The use of this technology consists of three stages: digitalization of the prosthetic preparation, virtual planning and production of the restoration. The dental preparation can be digitized outside the oral cavity, on the plaster model, or inside the oral cavity, through an intraoral scanning system. After scanning the preparation, the image will be transferred to a computer-aided design program (CAD), through which one the operator will be able to design the restoration in a virtual way. Through a milling machine or 3D digital printing, the CAM system will reproduce the desired rehabilitation. Depending on the structure and its purpose, you can choose from different materials, such as acrylic, ceramic, hybrid, metal, and $3 D$ resins. The objective of this work is to explain the use of the CAD / CAM system in Dentistry for the manufacture of fixed prostheses through a searching in the data bases Lilacs, Pubmed, Scielo and Google Academic. It was concluded that the CAD / CAM technology increases the versatility, precision and cost-benefit of making indirect restorations. It is believed that soon, it will be part of the clinical routine of most dentists.
\end{abstract}

KEYWORDS: Computer Aided Design. Dental prosthesis. Permanent dental restoration. 


\section{Introdução}

A evolução da tecnologia digital está transformando o cotidiano na Odontologia, principalmente na Reabilitação Oral. Quando se fala em prótese dentária pode-se notar grandes avanços, como por exemplo, a utilização do sistema CAD/CAM, derivado do inglês "Computer Aided Design - Computer Aided Manufacturing", significando Desenho Auxiliado por Computador/Usinagem Auxiliada por Computador ${ }^{1}$.

O seu uso na Odontologia conta inicialmente com uma ferramenta de digitalização do preparo protético que criará o modelo na tela do computador, possibilitando através desse modelo virtual, o planejamento da restauração¹. Após essa etapa, o processo CAM (ou processo de fresagem), irá materializar o desenho virtual da restauração que foi trabalhada no CAD (processo de desenho através de computadores). Fresadoras controladas por computadores realizarão os procedimentos de usinagem com alta precisão através de uma lista de movimentos que foi escrita em um código específico que irá permitir o controle de vários eixos para corte do material, assim os cuidados da usinagem são controlados².

Os progressos desses sistemas computadorizados para a produção de restaurações dentais associado ao desenvolvimento de novos materiais causaram uma mudança importante no fluxo de trabalho clínico para dentistas e técnicos, como também as opções de tratamento oferecidas ao paciente ${ }^{3}$. Devido a automatização no processo de planejamento e na produção das restaurações dentárias, alguns procedimentos laboratoriais deixaram de ser realizados em comparação às técnicas convencionais ${ }^{4}$.

A utilização do sistema CAD/CAM nos dias atuais, pode ser considerada uma poderosa ferramenta no âmbito protético, tendo em vista a forma como auxilia dentistas e laboratórios em todo o mundo. Estas restaurações apresentam algumas vantagens comparado as técnicas convencionais. O tempo de fabricação é reduzido, possui maior controle da espessura do material restaurador através de precisas mensurações, como também os materiais restauradores apresentam maior qualidade ${ }^{5}$.
Atualmente, a informatização tem trazido um grande suporte a Odontologia e os sistemas CAD/CAM trouxeram uma grande importância na reabilitação oral, pois já são realidade em muitos laboratórios de prótese e clínicas odontológicas. A implantação da Odontologia digital possui algumas dificuldades, como a curva de aprendizagem dos profissionais para as novas tecnologias, novos materiais e o seu alto investimento. Através do contexto apresentado, o objetivo primário do presente trabalho é explanar o uso do sistema CAD/CAM na Odontologia para confecção de próteses fixas. O objetivo secundário é discutir suas vantagens e as limitações deste novo fluxo de trabalho na Odontologia.

\section{Metodologia}

As buscas dos artigos da presente revisão de literatura foram realizadas nos idiomas português e inglês, no período entre março de 2019 até julho de 2020, e realizadas nas bases de dados Lilacs, Pubmed, Scielo, Google Acadêmico, além de consulta bibliográfica. Os descritores utilizados para a seleção dos artigos foram "Projeto auxiliado por computador"; "Prótese dentária"; "Restauração Dentária Permanente", em português e "Computer Aided Design"; "Dental Prosthesis"; Dental Restoration, Permanent", em inglês. Os artigos foram pré-selecionados a partir da leitura e análise dos títulos e respectivos resumos respeitando a linha de tempo de 2010 a 2020, porém foram coletados artigos mais antigos como os de 1971, 1988, 2005, 2006, 2009, pois traziam informações importantes sobre o CAD/CAM, sendo excluídos os materiais que não se adequavam ao estudo em relação ao conteúdo. Os artigos selecionados possuíam como abordagem principal o uso do sistema CAD/CAM na confecção de prótese fixa.

Além de artigos, foi utilizado o livro: CAD/CAM no laboratório e na clínica: Odontologia digital. Foram encontrados 53 artigos como base literária para realização desta revisão, porém foram utilizados 33 artigos, excluindo os 20 artigos os quais o conteúdo não era relevante sobre o tema. 


\section{Revisão de literatura}

O sistema CAD/CAM, é uma tecnologia bastante utilizada em várias indústrias e obteve a sua introdução na Odontologia no final da década de 70 e início da década de 80, através de Bruce Altschuler, nos EUA, François Duret, na França, Werner Mormann e Marco Brandestini, na Suíça. Tendo como objetivo, a automatização de processos manuais e padronização dos $\operatorname{mesmos}^{6,7}$.

O uso desse sistema está sendo utilizado na Odontologia para a produção de restaurações de prótese fixa, como por exemplo, inlays, onlays, coroas, prótese sobre implante e facetas. Várias empresas têm desenvolvido sistemas CAD-CAM de alta tecnologia baseados em três componentes: sistema de leitura da preparação dentária, o escâner, sistema de desenho da restauração protética auxiliado por computador (CAD) e o sistema de fresagem da estrutura protética (CAM) $)^{6,8}$.

Dentro da tecnologia CAD/CAM, existe uma gama de sistemas, como por exemplo, o sistema Cerec, sendo seguido do desenvolvimento de outros, como o Procera, Kavo Everest e o sistema Lava. O Cerec foi o primeiro sistema CAD/CAM que alcançou sucesso clínico e comercial. O primeiro modelo foi lançado no mercado em 1985 e após melhorias técnicas, outros modelos foram surgindo 9,10 . Correia et al. 6 apontam que o sistema Cerec é o único que apresenta duas versões, a de utilização clínica chamado de Cerec Chairside e a versão de laboratório. Segundo Abdullah et al. 9 o Cerec pode ser utilizado para trabalhos como inlays, onlays, coroas, prótese sobre implantes e próteses fixas parciais tendo a capacidade de escânear arcos superiores e inferiores.

Através da tecnologia do sistema Procera, hoje em desuso, a digitalização do modelo de gesso era feita por contato, por meio de um escâner Procera. A imagem digitalizada (3D CAD) era então enviada para uma central de processamento Procera ${ }^{\circledR}$ (Suécia Karlskoga e Estocolmo; E.U.A. - Nova Jersei) através de uma ligação por modem, onde eram efetuadas réplicas do modelo de gesso mais alargadas, de modo a compensar a contração da cerâmica quando da sua sinterização ${ }^{6}$.
O Kavo Everest é um sistema que inclui uma máquina de digitalização, um software CAD, uma máquina para fresagem e um forno para sinterizar a cerâmica. A digitalização do modelo de gesso será feita por uma leitura óptica através de uma câmara CCD (dimensão real 1:1 e precisão de $20 \mu \mathrm{m}$ ), assim sendo criada a imagem 3D através de 15 sequências de projeção. $A$ restauração protética será desenhada em um software CAD e logo após será fresada seguindo movimentos de corte de cinco eixos, em blocos de vários tipos de materiais, como por exemplo a zircônia parcialmente sinterizada - ZS -Blanks e a zircônia totalmente sinterizada - ZH - Blanks ${ }^{6}$.

O sistema Lava obteve a sua introdução dentro dos sistemas em 2002, sendo utilizado para fabricação de estrutura de zircônia para todas as restaurações de cerâmica. Este sistema utiliza bloco de policristais de zircônia tetragonal estabilizada com ítrio (Y-TZP), apresentando maior resistência à fratura do que cerâmicas convencionais. Utilizando um sistema óptico a laser para digitalizar as informações. O software CAD Lava localizará automaticamente a margem do preparo e sugerirá um pôntico. O CAM produz uma estrutura ampliada para compensar o encolhimento e um bloco de zircônia parcialmente sinterizado é selecionado para fresamento ${ }^{10}$.

Segundo Neves et al. ${ }^{11}$ através do sistema CAD/CAM, atualmente, diferentes fluxos de trabalho podem ser apresentados para a confecção de prótese fixa, como por exemplo: Possibilidade 1 - Obtenção de um escâner ("máquina de moldar"), tendo como objetivo agilizar o procedimento de moldagem e entrega do molde ao laboratório, trazendo também mais conforto ao paciente ao realizar esse procedimento; Possibilidade 2 - Obtenção do escâner e do software de desenho. Após o escaneamento o cirurgião-dentista poderá desenhar o término cervical dos preparos e acertar os contatos proximais antes de enviar ao laboratório; Possibilidade 3 - Obtenção de escâner, do software de desenho e da fresadora. A diferença desse fluxo de trabalho para o segundo, é a obtenção da fresadora, equipamento com o qual materializará o projeto realizado, embora dependa do laboratório de prótese para cristalização e maquiagem de alguns casos específicos; Possibilidade 4 - Obtenção do escâner, do software de desenho, fresadora e forno para cristalização, maquiagem e glaze de cerâmicas. 
Com esta opção o cirurgião-dentista poderá finalizar casos em resina e algumas cerâmicas no próprio consultório; Possibilidade 5 - Obtenção do escâner, do software de desenho, fresadora e fornos, para cristalização, maquiagem e glaze de cerâmicas vítreas e para sinterização de peças em zircônia. Podendo obter próteses fixas de até quatro elementos em zircônia, confeccionadas em consultório, tendo como objetivo agilizar a conclusão das peças.

Segundo Hilgert et al. ${ }^{1}$ para se obter um modelo virtual que irá reproduzir o preparo protético, realizado na boca do paciente, na tela do computador, pode-se optar por duas técnicas: a técnica de impressão óptica, através de um escâner intraoral ou através da impressão convencional, pela modelagem em gesso e digitalização do modelo através de um escâner de bancada (escâner extra oral). O escaneamento é uma técnica que realiza a digitalização de objetos a partir de imagens geradas por luz ou por contato². Uma das grandes vantagens da impressão digital utilizando o escâner intra-oral inclui a eliminação de etapas convencionais de moldagem, consequentemente eliminando possíveis distorções dos materiais de moldagem sendo uma vantagem também a sua efetividade em pacientes que possuem ânsia de vômito. Além disso os dados do paciente podem ser transmitidos ao laboratório utilizando-se a internet, reduzindo o risco de quebrar o modelo durante o seu transporte ${ }^{12}$.

Os escâneres intraorais comercialmente disponíveis se apresentam de duas maneiras: câmeras de imagem única ou de tecnologia fotográfica sendo elas, Itero (Align Technology), o PlanScan (Planmeca), o CS 3500 (Carestream Dental LLC) e o Trios (3 Shape) e as câmeras de vídeo ou tecnologia de vídeo, que são utilizadas pelo scanner True Definition (Lava Chairside Oral Scanner), Apollo DI (Dentysply - Sirona) e o sistema Omnicam System e Primescan (Dentysply Sirona). As câmeras de imagem única registram apenas uma secção, sendo necessária a sobreposição de imagens pelo software para a geração de um modelo digital 3D. Os escâners de tecnologia de vídeo operam de forma muito semelhante a uma câmera de vídeo, gravando assim as áreas escaneadas ${ }^{13}$.

Abduo et al. ${ }^{14} \mathrm{em}$ um trabalho de revisão sistemática observou que em comparação com as impressões convencionais, os sistemas de escaneamento intraorais podem ser usados de forma confiável para fins de diagnóstico e escaneamento de áreas curtas.
No entanto, para varredura de arco inteiro, o escaneamento intraoral é mais suscetível a erros. Os estudos analisados por eles indicaram resultados variáveis dos diferentes escaners intraorais. Embora a precisão dos escaners intraorais pareça promissora e comparável aos métodos convencionais, eles ainda são vulneráveis a imprecisões. Para a fabricação da prótese, a precisão do escanemanto intraoral é aprimorada reduzindo a extensão do escaneamento e garantindo que as superfícies escaneadas exibam irregularidades mínimas. Bohner et al. ${ }^{15}$ também em uma revisão sistemática estimou a precisão média das tecnologias digitais usadas para digitalizar tecidos faciais, esqueléticos e intraorais e corroborando com Abduo et al. ${ }^{14}$ concluiu que as tecnologias digitais atuais são consideradas precisas para aplicações específicas. No entanto, a digitalização de arcos completos ou edêntulos ainda representa um desafio.

Renne et al. ${ }^{16}$ avaliaram e compararam a velocidade, exatidão e precisão de 6 scanners intra-orais (CEREC Omnicam, CEREC Bluecam, Planscan, iTero, Carestream 3500, 3Shape) e um extra-oral (3Shape D800) em cenários de escaneamento de sextante e arco completo. Concluíram que todos os scanners avaliados diferem em relação a velocidade, precisão e exatidão, sendo que o Planscan e CEREC Omnicam fornecem melhor combinação desses fatores no cenário do escaneamento dos sextantes e o 3Shape no cenário de escaneamento de arco completo.

De acordo com Bernardes et al. $\_$o processo CAM, conhecido também como manufatura auxiliada por computador, é a etapa de reprodução da restauração trabalhada no software CAD. Hilgert et al. ${ }^{1}$ afirma que esse processo pode ser classificado de acordo com o local em que essa etapa acontece, dentro do próprio consultório, conhecido como "Chairside", no laboratório de prótese ou em algum centro de produção. Conforme Galhano et al.17 os sistemas CAD/CAM também podem ser classificados de acordo com a sua capacidade de compartilhar dados, podendo ser considerados como sistemas abertos ou fechados. Os sistemas fechados, restringem o profissional à dependência de um fabricante específico para todas as atualizações de software. Já o sistema aberto, permite a escolha entre diferentes combinações de CAD e CAM. No entanto, a tendência atual é que todos os sistemas sejam abertos. 
Nos últimos anos a digitalização ganhou uma grande importância na área odontológica, em particular dentro do fluxo de trabalho para prótese fixa ${ }^{18}$ e entre as vantagens desses sistemas, destacam-se a possibilidade de tratamento protético em sessão única, redução do número de etapas, menor tempo de confecção, a melhor reprodutibilidade e precisão dimensional dos preparos, arquivamento digital dos modelos e planejamento e a possibilidade de utilização de novos sistemas cerâmicos mais resistentes. Através do escaneamento é possível visualizar os preparos em uma tela com ampliação, possibilitando detectar áreas que necessitam de ajustes permitindo o controle de qualidade a nível micrométrico? ${ }^{2}$. Consequentemente, é possível obter coroas com maior grau de precisão na adaptação marginal.

Segundo Monteiro et al. ${ }^{19}$ uma má adaptação da prótese ocasionará retenção de placa e quanto maior for a discrepância marginal, mais rápida será a taxa de dissolução do cimento, causando inflamação dos tecidos. Mc Lean et al. 20 observaram que restaurações com gaps marginais até $120 \mu \mathrm{m}$ apresentam sucesso, sendo o limiar clinicamente aceitável preconizado na literatura. Para discrepância marginal no sistema CAD/CAM, preconiza-se de 50 a $100 \mu \mathrm{m}^{21-23}$. Os gaps marginais encontrados para coroas de resina confeccionadas com CAD/CAM intra-oral variam de 52 a $61 \mu \mathrm{m}^{24}$ para as coroas confeccionadas com materiais cerâmicos a partir do escaneamento intra-oral de 48 a $66^{24-26}$ e para o extra-oral variam de 53 a $111 \mu \mathrm{m}^{27,28}$.

Como toda tecnologia, os sistemas CAD/CAM possuem algumas limitações e dentre elas a que mais se destaca é a questão do custo, pois varia de acordo com as condições econômicas de cada país, como também o fluxo de pacientes de cada dentista ou laboratório, o que deve ser observado antes de adquirir esse equipamento ${ }^{1}$. Outra limitação do CAD/CAM está relacionada à necessidade de conhecimento do operador quanto ao uso de alguns softwares e hardwares para desenho das restaurações e o manuseio do equipamento de escaneamento ${ }^{2}$. O uso destas ferramentas possui uma curva de aprendizado e exigem dedicação do usuário.

Tendo em mente o tipo de restauração que irá ser realizada, o material que será utilizado e que diferentes parâmetros de fresagem como o tamanho da broca, afetam bastante o ajuste interno da restauração definitiva, qualquer preparo destinado a restaurações CAD/CAM, deve fornecer espaço adequado para o material e os seus ângulos devem ser arredondados, pois ângulos vivos e espaços inadequados podem levar a fraturas. Dentro de alguns softwares existem ferramentas especificas para detectar erros na preparação do dente ${ }^{29}$.

Os requisitos fundamentais para materiais restauradores usados para a confecção de coroas pelo sistema CAD/CAM são: boa usinabilidade, resistência à fratura e mínimos procedimentos de acabamento ${ }^{11}$. Os materiais disponíveis são resina acrílica e PMMA para confecção de provisórios e para restaurações definitivas a resina composta e cerâmica ${ }^{30,31}$.

A confecção de uma restauração provisória é de extrema importância, pois adequará o tratamento proposto nas questões de função e estética, alguns exemplos desses materiais são: Artegral ImCrown (Merz, Alemanha); Vita CAD-Temp (Vita, Alemanha); artBloc Temp (Merz); ZENO PMMA (Wieland, Alemanha); etkon polycon ae (Etkon, Alemanha) ${ }^{5}$.

Para confecção de restaurações definitivas, existe uma geração mais recente de blocos de resina composta, como por exemplo: Lava Ultimate (3M ESPE) e Cerasmart (GC) que apesar do nome, não são cerâmicas, e sim resinas densamente preenchidas com partículas polimerizadas a alta temperatura e pressão ${ }^{30,32}$. Outros blocos de resina atuais que também estão sendo utilizados são: Gradia Block (GC), Block HC (SHOFU INC.), KZR-CAD Hybrid Resin Block (Yamamoto Precious Metal), Grandio Blocs (VOCCO) ${ }^{33}$.

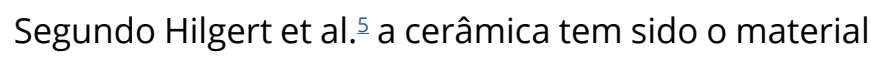
mais incorporado ao princípio de produção automatizada. Possuindo um alto padrão estético, juntamente com sua compatibilidade biológica e manutenção de lisura superficial e do brilho, algumas das vantagens que a cerâmica apresenta em relação a outras opções restauradoras.

Os materiais cerâmicos disponíveis são: cerâmicas feldspáticas; cerâmicas vítreas reforçadas por cristais de leucita; e vítreas à base de cristais de dissilicato de lítio, a zircônia, o silicato de lítio reforçado por zircônia. As cerâmicas feldspáticas são as mais utilizadas por apresentarem uma característica importante como a não necessidade de ir ao forno e facilidade de polimento ${ }^{5}$. Os representantes das cerâmicas feldspáticas são VITABLOCS Mark II (VITA 
Zahnfabrik), VITABLOCS RealLife, Ceramic Blocks (VITA), and VITABLOC TriLux Forte (VITA). Com maior resistência em comparação a cerâmica feldspática, a cerâmica de vidro reforçada com leucita é indicada especialmente para coroas anteriores, inlays/onlays e coroas posteriores ${ }^{29}$.

Cerâmicas vítreas à base de dissilicato de lítio apresentam resistência flexural biaxial de aproximadamente $360 \mathrm{Mpa}$, sendo assim indicadas para produção de inlays/onlays, coroas anteriores e posteriores, copings para coroas unitárias ou infraestruturas para pontes de até três elementos no segmento anterior ${ }^{5}$.

Um bloco de material cerâmico que pode ser citado é o Vita Enamic (Vita Zahnfabrik), o qual é uma cerâmica híbrida, sendo uma combinação da cerâmica feldspática enriquecida com óxido de alumínio. Esse material além de uma grande elasticidade, possui uma enorme resiliência após a união adesiva combinando as propriedades positivas da cerâmica e do compósito ${ }^{30}$.

Outro material o qual o uso foi potencializado pela utilização das tecnologias, é o dióxido de zircônio ou também conhecido como zircônia. Seus pontos principais como material para coroas monolíticas e criação de infraestruturas, é a sua elevada resistência flexural, excelente biocompatibilidade e na era da estética, sua coloração e translucidez trazem ótimos resultados estéticos ${ }^{5}$. Podem ser citados também o silicato de lítio reforçado com óxido de zircônio (ZLS), a nova geração de cerâmica vítrea de alta resistência, utilizando o sistema Celtra Duo (Dentsply Sirona). Assim como a cerâmica de silicato de lítio, que se tornou popular por várias indicações, como para coroas monolíticas, inlays e onlays ${ }^{29}$.

\section{Considerações finais}

Com base nos dados que foram encontrados na literatura cientifica, pode-se chegar à conclusão que a evolução dos sistemas CAD/CAM utilizados atualmente na área odontológica, são capazes de reproduzir restaurações protéticas de alta qualidade com diferentes opções de materiais restauradores e tipos de próteses. O sistema CAD/CAM está cada vez mais presente nos consultórios e laboratórios de prótese e como consequência grande parte dos tratamentos reabilitadores podem ser planejados e executados do seu início ao fim de maneira digital. O uso da tecnologia CAD/CAM para a confecção de prótese fixa tem se mostrado vantajoso por conjugarem estética, precisão, durabilidade, facilidade de execução, conforto e agilidade do tratamento, tanto para o profissional quanto para o paciente. No entanto, o aporte financeiro para a implementação da tecnologia e treinamento da equipe é alto. Existem muitos sistemas digitais disponíveis atualmente na área odontológica, cabe ao cirurgião-dentista e ao laboratório de prótese escolher qual se adequa melhor à sua rotina.

\section{Contribuições das autoras}

Borges LO contribuiu para coleta de dados bibliográficos e escrita do presente trabalho. Carvalho $\mathrm{AO}$ realizou a orientação e supervisão do trabalho, a escrita e revisão do manuscrito. Lima EMC contribuiu com a escrita e revisão do manuscrito.

\section{Conflitos de interesses}

Nenhum conflito financeiro, legal ou político envolvendo terceiros (governo, empresas e fundações privadas, etc.) foi declarado para nenhum aspecto do trabalho submetido (incluindo, mas não se limitando a subvenções e financiamentos, participação em conselho consultivo, desenho de estudo, preparação de manuscrito, análise estatística, etc.).

\section{Referências}

1. Hilgert LA, Schweiger J, Beuer F, Andrada MAC, Araújo E, Edelhoff D. Odontologia Restauradora com Sistemas CAD / CAM : o Estado Atual da Arte Parte I - Princípios de Utilização. Clín Int J Braz Dent. 2009;5(3):294-303.

2. Bernardes SR, Tiossi R, lam S, Thomé G. Tecnologia CAD / CAM aplicada a prótese dentária e sobre implantes: o que é, como funciona, vantagens e limitações: uma revisão crítica da literatura. Ilapeo. 2012;06(01):8-13.

3. Silva LH , Lima E, Miranda RBP, Favero SS, Lohbauer U, Cesar PF. Dental ceramics: a review of new materials and processing methods. Braz Oral Res. 2017;31(Suppl 1):133-45. http://dx.doi. org/10.1590/1807-3107BOR-2017.vol31.0058

4. Hilgert LA, Schweiger J, Beuer F, Andrada MAC, Araújo E, Edelhoff D. Odontologia Restauradora com Sistemas CAD / CAM : o Estado Atual da Arte Parte 2 - Possibilidades Restauradoras e sistemas CAD/CAM. Int J B Dent. 2009;5(4):424-435. 
5. Hilgert LA, Schweiger J, Beuer F, Andrada MAC, A Élito. Odontologia Restauradora com Sistemas CAD / CAM : O Estado Atual da Arte Parte 3 - Materiais Restauradores para sistemas CAD/CAM. Int J B Dent. 2010;6(1):86-96.

6. Correia ARM, Fernandes JCAS, Cardoso JAP, Leal C, Silva DA. CAD-CAM : a informática a serviço da prótese fixa. Revista de Odontologia da UNESP. 2006;35(2):183-9.

7. Duret $F$, Blouin LJ, Duret B. CAD-CAM in dentistry. JADA. 1988;117(6):715-20. http://dx.doi.org/10.14219/jada. archive.1988.0096

8. Liu PR. A Panorama of Dental CAD/CAM Restorative Systems. Compend Contin Educ Dent. 2005;26(7):507-8.

9. Abdullah AO, Muhammed FK, Zheng B, Liu Y. An Overview of Computer Aided Design / Computer Aided Manufacturing (CAD/ CAM) in Restorative Dentistry. J Dent Mater Tech. 2017;7(1):1-10.

10. Mantri SS, Bhasin AS. Cad/CAM In Dental Restorations: An Overview. Ann Essences Dent. 2010;2(3):123-8.

http://dx.doi.org/10.5368/aedj.2010.2.3.123-128.pdf

11. Neves FD, Prado CJ, Karam FK, Resende CC, Tavares LC, Carneiro TAP et al. Experiência de Cinco Anos de Utilização do Sistema CEREC - Benefícios e Dificuldades Encontradas. In: Pádua MJ, Teles RF. CAD/CAM No Laboratório e Na Clínica: a Odontologia Digital. São Paulo: Napoleão editora, 2017. P.302-23.

12. Kihara H, Hatakeyama W, Komine F, Takafuji K, Takahashi T, Yokota J et al. Accuracy and practicality of intraoral scanner in dentistry: A literature review. J Prosthodont Res. 2019;64(2):10913. http://dx.doi.org/10.1016/j.jpor.2019.07.010

13. Alghazzawi TF. Advancements in CAD / CAM technology: Options for practical implementation. J Prosthodont Res. 2016;60(2):72-84. http://dx.doi.org/10.1016/j.jpor.2016.01.003

14. Abduo J, Elseyoufi M. Accuracy of Intraoral Scanners: A Systematic Review of Influencing Factors. Eur J Prosthodont Restor Dent. 2018;26(3):101-121. http://dx.doi.org/10.1922/ EJPRD 01752Abduo21

15. Bohner L, Gamba DD, Hanisch M, Marcio BS, Tortamano Neto $P$, Laganá DC et al. Accuracy of digital technologies for the scanning of facial, skeletal, and intraoral tissues: A systematic review. J Prosthet Dent. 2019;121(2):246-51. http://dx.doi. org/10.1016/j.prosdent.2018.01.015

16. Renne W, Ludlow M, Fryml J, Schurch Z, Mennito A, Kessler $R$ et al. Evaluation of the accuracy of 7 digital scanners: An in vitro analysis based on 3-dimensional comparisons. J Prosthet Dent. 2017;118(1):36-42. http://dx.doi.org/10.1016/j. prosdent.2016.09.024
17. Galhano GAP, Pellizzer EP, Mazaro JVQ. Optical impression systems for CAD-CAM restorations. J Craniofac Surg. 2012;23(6):e575-9. http://dx.doi.org/10.1097/ $\underline{\mathrm{SCS} .0 \mathrm{~b} 013 \mathrm{e} 31826 \mathrm{~b} 8043}$

18. Schmidt A, Klussmann L, Wöstmann B, Schlenz MA. Accuracy of Digital and Conventional Full-Arch Impressions in Patients : An Update. J Clin Med. 2020;688(9): 1-9. http://dx.doi.org/10.3390/ jcm9030688

19. Monteiro TC, Franco CB, Silva LB, Araújo MA, Chagas RB, Saldanha ADD et al.. Sistema CAD / CAM para confecção de próteses dentárias. Revista Diálogos Acadêmicos. 2019;8(2):31-5.

20. McLean JW, von Fraunhofer JA. The estimation of cement film thickness by an in vivo technique. Br Dent J. 1971;131(3):107-11. http://dx.doi.org/10.1038/sj.bdj.4802708

21. Ural C, Burgaz Y, Saraç D. In vitro evaluation of marginal adaptation in five ceramic restoration fabricating techniques. Quintessence Int. 2010;41(7):585-90.

22. Euán R, Figueras-Álvarez O, Cabratosa-Termes J, Brufaude Barberà M, Gomes-Azevedo S. Comparison of the Marginal Adaptation of Zirconium Dioxide Crowns in Preparations with Two Different Finish Lines. J Prosthodont. 2012;21(4):291-5. http:// dx.doi.org/10.1111/j.1532-849x.2011.00831.x

23. Euán R, Figueras-Álvarez O, Cabratosa-Termes J, OliverParra R. Marginal adaptation of zirconium dioxide copings: Influence of the CAD/CAM system and the finish line design. J Prosthet Dent. 2014;112(2):155-62. http://dx.doi.org/10.1016/j. prosdent.2013.10.012

24. Silveira ACP, Chaves SB, Hilgert LA, Ribeiro APD. Marginal and internal fit of CAD-CAM-fabricated composite resin and ceramic crowns scanned by 2 intraoral cameras. J Prosthet Dent. 2017;117(3):386-92. http://dx.doi.org/10.1016/j. prosdent.2016.07.017

25. Neves FD, Prado CJ, Prudente MS, Carneiro TAPN, Zancopé $\mathrm{K}$, Davi LR et al. Micro-computed tomography evaluation of marginal fit of lithium disilicate crowns fabricated by using chairside CAD/CAM systems or the heat-pressing technique. J Prosthet Dent. 2014;112(5):1134-40. http://dx.doi.org/10.1016/j. prosdent.2014.04.028

26. Ng J, Ruse D, Wyatt C. A comparison of the marginal fit of crowns fabricated with digital and conventional methods. J Prosthet Dent. 2014;112(3):555-60. http://dx.doi.org/10.1016/j. prosdent.2013.12.002

27. Meirowitz A, Bitterman Y, Levy S, Mijiritsky E, Dolev E. An in vitro evaluation of marginal fit zirconia crowns fabricated by a CAD-CAM dental laboratory and a milling center. BMC Oral Health. 2019;19(1):1-6. http://dx.doi.org/10.1186/s12903-019-0810-9 
28. Alqahtani F. Marginal fit of all-ceramic crowns fabricated using two extraoral CAD/CAM systems in comparison with the conventional technique. Clin Cosmet Investig Dent. 2017;9:13-8. http://dx.doi.org/10.2147/CCIDE.S130856

29. Blatz MB, Conejo J. The Current State of Chairside Digital Dentistry and Materials. Dent Clin North AM. 2019;63(2):175-97. http://dx.doi.org/10.1016/j.cden.2018.11.002

30. Sulaiman TA. Materials in digital dentistry-A review. J Esthet Restor Dent. 2020;32(1):171-81. http://dx.doi.org/10.1111/ jerd.12566
31. Saeed F, Muhammad N, Khan AS, Sharif F, Rahim A, Ahmad $P$ et al. Prosthodontics dental materials: From conventional to unconventional. Mater Sci Eng C Mater Biol Appl. 2020;106:110167. http://dx.doi.org/10.1016/j.msec.2019.110167

32. Curran P, Cattani-Lorente M, Wiskott HWA, Durual S, Scherrer SS. Grinding damage assessment for CAD-CAM restorative materials. Dent Mater. 2016;33(3):294-308. http://dx.doi. org/10.1016/j.dental.2016.12.004

33. Kamonkhantikul K, Arksornnukit M, Lauvahutanon $\mathrm{S}$, Takahashil $\mathrm{H}$. Toothbrushing alters the surface roughness and gloss of composite resin CAD/CAM blocks. Dent Mater J. 2016;35(2):225-32. http://dx.doi.org/10.4012/dmj.2015-22 